\title{
Barriers for User Acceptance of Mobile Health Applications for Diabetic Patients: Applying the UTAUT Model
}

\author{
Fazlyn Petersen $^{(\mathbb{})} \mathbb{( D}$, Mariam Jacobs (D), and Shaun Pather (D) \\ University of the Western Cape, Bellville, South Africa \\ \{fapetersen, spather\}@uwc.ac.za, 3462668@myuwc.ac.za
}

\begin{abstract}
The literature illustrates that technology will widen health disparity if its use is restricted to patients who are already motivated and demonstrate good self-management behaviours. Additionally, despite the availability of free mobile health (m-health) applications for diabetes self-management, usage is low. There are also limited studies of m-health acceptance in South Africa. This research is delineated to the Western Cape, South Africa. The populace suffers from increasing numbers of diabetic patients. Segments of the population also suffer from technological forms of exclusion, such as limited internet access. Therefore, the objective of this study was to identify challenges for user acceptance that discourages the use of m-health applications. This study analysed 130 semi-structured interviews, using thematic content analysis. Respondents were predominantly female with type 2 diabetes, older than 50, residing in the Western Cape. It used key constructs from the Unified Theory of Acceptance and Use of Technology (UTAUT) model. The results confirmed that all four UTAUT constructs; performance expectancy ("the degree to which an individual believes that using the system will help him or her to attain gains in performance"), effort expectancy ("the degree of ease associated with the use of the system", social influence ("the degree to which an individual perceives that important others believe he or she should use the new system") and facilitating conditions ("the degree to which an individual believes that an organisational and technical infrastructure exists to support the use of the system"), explains the challenges for m-health acceptance in low socio-economic areas. Factors such as technology anxiety, resistance to change and a lack of trust in the use of devices for self-management need to be considered when implementing future interventions.
\end{abstract}

Keywords: Unified Theory of Acceptance and Use of Technology (UTAUT) Challenges for user acceptance $\cdot$ Mobile health (m-health) $\cdot$ Diabetes self-management $\cdot$ Low socio-economic areas $\cdot$ South Africa

\section{Introduction}

The use of technology is warranted due to the increasing number of patients with diabetes, especially in Low- and Middle-Income Countries (LMIC). Diabetes is the leading cause of mortality, of which $80 \%$ of deaths occur in LMIC [1]. Many of the 
deaths directly attributable to diabetes occur before the age of 70 [2]. Low socioeconomic status has been associated with the prevalence of type 2 diabetes [3, 4]. Research indicates that there are inequalities in diabetes control amongst "racial/ethnic minorities and those with low socioeconomic status" [4].

Therefore, the Western Cape (WC), a province in South Africa, provides the geographical area where challenges for use can be studied. This is due to the fact that $10.4 \%$ of the WC population has succumbed to diabetes [5]. Type 2 diabetes mellitus (T2DM) is the leading underlying cause of death in women (7.2\%) and second amongst both genders and people of all ages [5]. It is forecasted that by the year 2040, one in every ten adults residing in LMICs will be living with diabetes [6]. Therefore, selfmanagement is an important part of the treatment in Non-Communicable Diseases (NCD) [7] such as diabetes. Diabetes self-management is crucial to ensure that longterm complications are decreased. Diabetes self-management is based on seven selfcare behaviours [8]. These include; healthy eating, being active, monitoring, taking medication, problem-solving, healthy coping, and reducing risks [8].

It is estimated that $49.2 \%$ of the South African adult population lives below the upper-body poverty line [9] with less than R 1183 (nearly \$79) per person per month [10]. Additionally, the digital divide remains evident as parts of this populace experience technology inequalities [11].

Research indicates that the use of smartphone technologies and mobile phone applications, such as mobile health (m-health), may facilitate diabetes self-management [12]. This is due to m-health being an interactive, inexpensive and dynamic means of supporting diabetes patients with self-care behaviours [13]. Thereby reducing mortality rates by delivering effective interventions to patients [14]. However, despite the availability of $\mathrm{m}$-health for diabetes self-management, the overall uptake of $\mathrm{m}$-health diabetes management was low [12] and continued use is low [15].

\section{Challenges for User Acceptance}

Models for user acceptance demonstrates that individuals' reaction to information technology drives their intention to use information technology, such as m-health. This then ultimately determines their actual use. Research indicates that an individuals' intention to use a system such as m-health may explain the actual use of information of a system [16] or alternatively can be used to explain the challenges when use behavior is low. For example, if using the information technology is slow and difficult to use, this may influence individuals to use it less frequently or abandoning the technology [16].

The UTAUT model is an established user acceptance model with eight models used to develop it, including the Theory of Reasoned Action, the Technology Acceptance Model and the Theory of Planned Behaviour, explained between $17 \%$ and $53 \%$ variance in user intentions to use technology [16]. The UTAUT model explained $77 \%$ of the variance in behavioural intention to use technology and $52 \%$ of the variance in technology use.

The UTAUT (Fig. 1) includes four core constructs (performance expectancy, effort expectancy, social influence and facilitating conditions) that are direct determinants of 


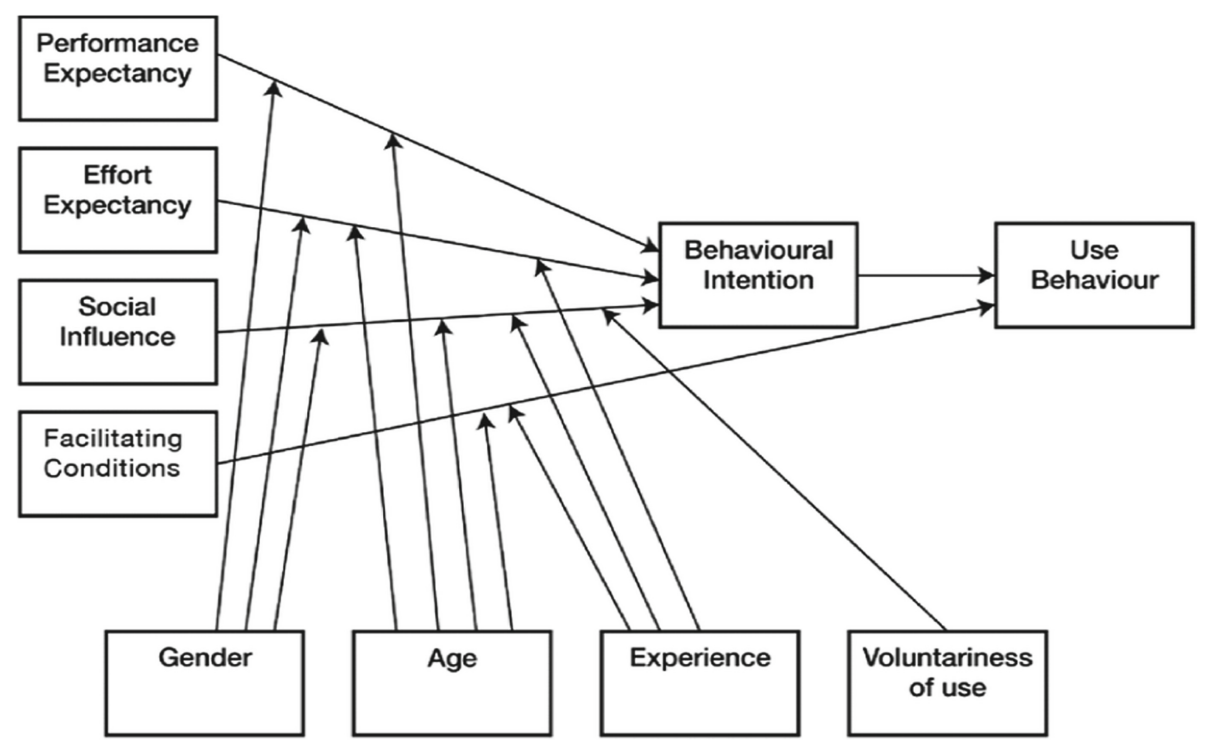

Fig. 1. Unified theory of acceptance and use of technology (Source: [16], p. 447)

behavioural intention and use. These constructs are moderated by gender, age, experience, and voluntariness of use [16].

This paper's authors have conducted a study on user acceptance of Information Communication and Technology (ICT) for diabetes self-management in the Western Cape, South Africa. This study also used the UTAUT model [16]. The research found that behavioural intention did not translate into usage [17]. This was due to the fact that almost $70 \%$ of respondents did not use forms of ICT, such as m-health applications, for their diabetes self-management. Disparities in use have been identified in the literature for older adults, low income and racial/ethnic minorities [18, 19]. Therefore, this paradox serves as the problem in this study, which focuses on analysing challenges to the acceptance and usage of mobile health applications.

Based on the areas identified by the study above [17], the objective of this study is to determine barriers for the acceptance of diabetes m-health applications, given the currently low levels of $m$-health use for diabetes self-management in the Western Cape.

The literature indicates that context may impede the acceptance and use of m-health applications [20]. Research already indicates mixed findings on reaching at-risk populations as most apps focus on high-cost populations [21], leaving unexplored the reach to the most at-risk population groups in South Africa, who will then remain disadvantaged because the actualisation of mobile phones for aiding service access will remain confined [22]. It is imperative to address the cost-effectiveness challenges inherent in implementation [22, 23]. Designers of interventions must recognise the constraints of the South African health system and consider appropriate options for this context. 


\subsection{Effort Expectancy}

For patients aged 50 years or older, effort expectancy was identified as a key factor for diabetes applications [24]. The positive impact of effort expectancy on behavioural intention is supported by other studies that analysed consumer usage of health informatics [25]. However, the impact of effort expectancy on behavioural intention is more prominent for older users as a study with younger and well-educated users found that this relationship was not significant [25]. Therefore, diabetes applications should be designed so that they are easy to use and understand, especially for older users.

\subsection{Performance Expectancy}

Research indicates that performance expectancy is an important determinant of the intention to use diabetes management apps [25]. The finding is supported by a study that indicates performance expectancy's significant impact on the users' behavioural intention to adopt mHealth services in Bangladesh [19].

Performance expectancy may be low as the perception of medical professionals is that technology use and data capture is a low-status activity. Hence, the task is delegated to junior personnel [26]. The negative perception and delegation may lead to user resistance [26].

\subsection{Social Influence}

Social influence is found to be a significant determinant of the intention to use diabetes management apps [25]. This finding is supported by the fact that supportive health care professionals and family members are core to m-health acceptance [27].

M-health data on applications, such as Glucose buddy, can be shared with health care providers. However, this may leave patients to feel vulnerable when their health care providers do not provide empathy or solutions when they are sharing their high glucose readings [28]. A lack of empathy and concern by the people who patients deem as important may lead to the discontinued use of $\mathrm{m}$-health applications.

\subsection{Facilitating Conditions}

Only $25.8 \%$ of Western Cape residents have access to the internet at home [29]. Additionally, $61.7 \%$ of people in the Western Cape have access to the internet using their mobile devices of which $26.8 \%$ of people in the rural have access to the internet [29]. With the increasing inequalities amongst different ethnicities, there are disparities between income, access, education and health literacy amongst LMICs. Subsequently, it may impact diabetes self-management as access to information is essential for successful self-management [30].

Additionally, South African m-health services are based on unsustainable business models due to the dependence on donor funding [22, 23]. There is a high risk for discontinuation of services. Therefore, there is a need for innovative business models that are based on best practice [31, 32]. 
There are limited information technology (IT) skills and training especially among older users [33]. Health apps are downloaded and used without guidance [34]. Therefore, to raise the level of acceptance among older users, allow for personal contact persons during the initial phase of use [24] and training.

\section{Methodology}

This research used an interpretivist paradigm [35]. Interpretivism highlights the view that reality is socially constructed and subjective [36]. Therefore, it is a suitable paradigm for this research seeks to interpret the perception of individuals' acceptance and use of m-health to identify barriers.

\subsection{Data Collection and Analysis}

This research used qualitative data collected from $26 \%$ of respondents used in the initial study, User acceptance of ICT for diabetes self-management in the Western Cape, South Africa [17]. The initial study analysed quantitative data from 498 respondents using an online survey. Respondents resided in predominantly low socioeconomic areas, such as Mitchell's Plain, Belhar, Khayelitsha, Athlone, Delft as well as Gugulethu, in the Western Cape. For this study, 130 diabetic respondents who participated in the initial study were selected using random sampling and semi-structured interviews [37]. Interviews were conducted by third-year Information Systems students working as field workers, using an interview guide.

Interview questions included the following to gain insight into the broader use of Information Communication and Technology (ICT), including m-health applications, as it could not be assumed that the lack of m-health application usage meant that no alternative ICT options were being used:

"Which technology (ICT) do you use to help manage your diabetes?

Prompt if required: Do you use a glucose testing machine? Do you search for diabetes-related information on the internet? Do you use an application on your mobile phone?

What prevents you from using ICT, including mobile applications, for managing your diabetes?

Prompt if required: If they don't use ICT such as mobile applications, ask them if it's related to cost or whether it's too difficult to use. Is it anything else?

Do you find ICT, including mobile applications, useful for managing your diabetes?

Prompt if required: Does it help you achieve better results when you go to the doctor?

What do you like or dislike about the current technology for managing your diabetes?

Prompt if required: Is it easy to use and understand? Is it easy to incorporate into your life?" [30].

The study used thematic content analysis, using Atlas.ti software. The data analysis was based on the key thematic areas identified in the theoretical framework (Fig. 1). The steps for thematic content analysis included the preparation, organisation and reporting of the transcribed interviews [38]. 


\subsection{Ethical Considerations}

The study was subjected to the protocols for ethical clearance by the Human and Social Sciences Research Ethics Committee at the University of the Western Cape. In this regard, a high level of attention was paid to observing the strictest levels of confidence during the data collection exercise. All respondents were advised of their rights and had to consent to participate in the research. It should be noted though, that the nature of the questions was restricted to issues of m-health acceptance and use, rather than on specifics of an individuals' personal and private health situation.

\section{Results}

The results commence with demographics and conclude with thematic content analysis findings.

\subsection{Demographics}

The demographics below indicate that the majority of the respondents are females (52\%). The predominant respondent age group is older than $50(36 \%)$ (Table 1). A limited number of respondents (17.69\%) used mobile health applications while the highest percentage $(30.76 \%)$ preferred to use glucose testing machines.

Table 1. Demographics

\begin{tabular}{l|l|r|l}
\hline \multicolumn{2}{c|}{} & Frequency & Percentage \\
\hline \multirow{4}{*}{ Gender } & Male & 55 & 42.31 \\
\cline { 2 - 4 } & Female & 67 & 51.54 \\
\cline { 2 - 4 } & Unknown & 8 & 6.15 \\
\cline { 2 - 4 } Age & Total & 130 & 100 \\
\cline { 2 - 4 } & Between 16 \& 24 & 10 & 7.69 \\
\cline { 2 - 4 } & Between 25 \& 34 & 17 & 13.08 \\
\cline { 2 - 4 } & Between 35 \& 49 & 43 & 33.08 \\
\cline { 2 - 4 } & Older than 50 & 47 & 36.15 \\
\cline { 2 - 4 } & Unknown & 13 & 10 \\
\cline { 2 - 4 } & Total & 130 & 100 \\
\hline
\end{tabular}

\subsection{Themes Identified from the UTAUT Model}

To reach the objective, themes were coded according to UTAUT constructs. It was found that all four constructs explain the challenges experienced for acceptance in low socio-economic areas. 


\subsubsection{Performance Expectancy}

Respondents indicated that they did not believe that using m-health applications would assist them to attain better health. This was highlighted by the following quotation:

"I don't see the purpose. I receive everything from my testing machine" and "I don't want to play with my health and get things wrong and end up making myself sick. I'm comfortable with the doctors handling the heavy work."

Older respondents use their mobile phones to make phone calls and thus their willingness to use m-health is lower. Respondents also mentioned that there is no need for $m$-health apps because their condition is manageable. This was summarised by the following quote "I don't use anything else because I don't see the need to. I have my family to help me, and if I need more info I will ask one of them to find out for me, or I will get the information when I go to the doctor again".

\subsubsection{Effort Expectancy}

Respondents indicated that they dislike using technology as it is too complex for older people as well as being too difficult for them to use. One respondent mentioned, "For the older generation, technology can be a bit complex to use".

Evidence shows that respondents that they are unable to complete self-management activities, using m-health. Respondents mentioned that using $\mathrm{m}$-health is intimidating to them. The intimidation is due to respondents having limited ICT experience and skills to use m-health. This was prevalent for older respondents and resulted in lower usage. Therefore, the design of the m-health application is seen as a challenge as patients do not have the ability to use the m-health on their own.

Furthermore, respondents identified that using m-health will be easier than using the traditional approach to seek medical consultation. Attending health care facilities is inconvenient for elderly patients as they will have to wait for hours or even a full day to be examined by a professional. Therefore, using $\mathrm{m}$-health will allow more time for other activities.

A respondent stated, "I really do not know, I take it from myself, it's difficult for me because my eyes are blurry". Thus, the interface should be user-friendly for older patients to incorporate into their daily lifestyles. Moreover, respondents use other tools such as glucose meters rather than m-health as it is easy to use and understand. Furthermore, individuals mentioned that operating m-health was not easy at first and after many attempts, it became easier. This is summarised by the following comments, "Found it challenging in the beginning" and "I struggled at first but I think I'm getting better now".

\subsubsection{Social Influence}

Respondents stated that family and friends encouraged $\mathrm{m}$-health usage to manage their diabetes. However, the results also indicated that respondents preferred the assistance and social support of family and friends as opposed to using a device for selfmanagement activities.

Respondents also indicated that having in-person consultations with healthcare professionals provides a more accurate representation of their illness than managing it 
using m-health. This is summarised by the following statements: "Feel like the doctor is more accurate at giving results" and "the doctor learns [teaches] you more".

\subsubsection{Facilitating Conditions}

Cost was identified as a determining factor for m-health acceptance across all the respondents in all age groups. This is due to data being too expensive for people residing in low resource areas to afford. This is a salient factor as many people prefer spending their funds on supporting their families with their basic needs as to spending on m-health. This was summarised by one respondent who stated that "They [m-health] are quite costly \& being a family man, it can be hard to afford".

Respondents stated that they have access to technology. Yet, it is mainly used for social media such as WhatsApp, Facebook and phone calls as opposed to diabetes selfmanagement. Despite having access to a cell phone, respondents do not have the necessary resources to download m-health applications as it requires certain software and data/WIFI to download, update and track the results on an application.

Interoperability was identified as a challenge for the acceptance of m-health. Respondents mentioned that m-health is not compatible with other systems, such as glucose testing machines. Therefore, in order for patients to monitor their selfmanagement activities, they have to use more than one ICT tool. Respondents mentioned that privacy and security is a concern. They fear that others can potentially obtain their personal information.

Lack of training is identified as a reason for the lack of use or the discontinuation of use. This further illustrates that respondents will accept and use m-health provided that a specific person is available for assistance with $\mathrm{m}$-health difficulties. Respondents indicated that they are unable to afford to pay for training. Respondents further indicated that when training is provided, they are not aware of it. Respondents mentioned that they "Don't have the right training for it", "People can't afford to learn" and "do not know when it [training] is available”.

\section{Implications for the Design of M-Health Interventions}

Practical approaches to privacy and security need to be implemented as patients are entering personal health information [39]. Personal contact persons, especially during the initial phase of use, are essential to reduce the fear of data loss or erroneous data input. This may raise acceptance among older patients [43]. Additionally, elderly patients should be included as part of the stakeholder group to design health applications so that the needs and limitations of the target user group into consideration [57]. This will result in m-health applications designed in a way that is easy to use and understand by older patients.

Results show that patients prefer social support from family and friends as opposed to seeking information from a mobile application. This may be linked to factors such as technology anxiety, resistance to change and a lack of trust in the use of devices for self-management. Therefore, interventions such as personal contact during the initial phase of use should be leveraged as this will allow the acceptance of use amongst patients, especially older adults [24]. Respondents stated that face-to-face consultations 
provide more accurate information than a self-management tool such as m-health applications. To overcome the challenge of inaccurate information provided by health applications, a single framework should be developed to evaluate the role of m-health and e-health tools in strengthening the health system.

Affordability and access to ICT are identified by authors as an important factor for acceptance and use for m-health [39-41]. Access as a barrier may be difficult to overcome as many of the WC population are living below the poverty line with limited device and internet access, failing to consider these factors may result in a limited reach of at-risk patients. Literature supports the view that training is necessary to improve usage for patients as well as health care staff [42, 43]. However, funding will be required to implement training initiatives and health care in the Western Cape has experienced significant downsizing of personnel as well as population growth exceeding funding growth [44]. Essentially, training among older users is required as participants mentioned that they would accept m-health application given that assistance is available for any m-health difficulties. To raise the level of acceptance among older users, personal contact persons should be available during the initial phase of use. Despite having access to technology, patients are unable to complete self-management activities on their own due to lack of resources to download m-health applications. Interventions should take into account the constraints of South African's health system and consider the use of open-source options [24, 45].

\section{Conclusion}

This study set out to investigate the barriers to user acceptance of m-health applications. Drawing on the literature, the UTAUT model was used as a basis to inform this study. In particular, the four key constructs of the UTAUT model, performance expectancy, effort expectancy, social influence and facilitating conditions were used to determine the barriers for user acceptance of mobile applications discourage use and prevent behavioural intention to be converted into use. The results indicate that all four constructs of the UTAUT model can explain the barriers for user acceptance of mhealth applications for diabetic patients in the Western Cape.

Despite the many barriers, the study has found that diabetic patients stated that using m-health will be easier than using the traditional approach to seek medical consultation. This is due to m-health applications being more convenient. Furthermore, diabetic patients mentioned that operating m-health became easier after many attempts. Therefore, interventions such as training should be implemented.

The barriers identified in this study is limited to diabetic patients residing in the Western Cape and may not be generalised to the entire South African population. Further research into the reasons for the lack of trust and not identifying a need to use $\mathrm{m}$-health, by patients in the low socio-economic areas in the Western Cape, is required. There may also be other challenges identified by using the themes from another acceptance model, such as the Innovation Diffusion Model [46]. 


\section{References}

1. World Health Organization: Noncommunicable disease. WHO (2015)

2. World Health Organisation: Diabetes (2018). http://www.who.int/news-room/fact-sheets/ detail/diabetes. Accessed 25 Nov 2018

3. Berkowitz, S., et al.: Low socioeconomic status is associated with increased risk for hypoglycemia in diabetes patients: the Diabetes Study of Northern California (DISTANCE). J. Health Care Poor Underserv. 25(2), 478-490 (2014)

4. Ruddock, J.S., Poindexter, M., Gary-Webb, T.L., Walker, E.A., Davis, N.J.: Innovative strategies to improve diabetes outcomes in disadvantaged populations. Diabet. Med. 33, 723-733 (2016)

5. Statistics South Africa: Mortality and causes of death in South Africa. Pretoria (2016)

6. Reid, M., et al.: Development of a health dialogue model for patients with diabetes: a complex intervention in a low-/middle income country. Int. J. Africa Nurs. Sci. 8(July), 122131 (2018)

7. Lunde, P., Nilaaon, B., Bergland, A., Kvaerner, K., Bye, A.: The effectiveness of smartphone apps for lifestyle improvement in noncommunicable diseases: systematic review and meta-analyses. J. Med. Internet Res. 20(5), e162 (2018)

8. American Association of Diabetes Educators: Self care behaviors. Diabetes Self Manag. 111 (1997)

9. Statistics South Africa: Living conditions of households in South Africa: an analysis of household expenditure and income data using the LCS 2014/2015. Statistical release P0310 (2017)

10. Statistics South Africa: National Poverty Lines 2018. Statistical release P0310, no. July, pp. 1-10 (2018)

11. Gillwald, A., Mothobi, O., Rademan, B.: After access paper series: the state of ICT in South Africa (2017)

12. Garabedian, L.F., Ross-Degnan, D., LeCates, R.F., Wharam, J.F.: Uptake and use of a diabetes management program with a mobile glucometer. Prim. Care Diabetes 13, 549-555 (2019)

13. Hou, C., Xu, Q., Diao, S., Hewitt, J., Li, J., Carter, B.: Mobile phone applications and selfmanagement of diabetes: a systematic review with meta-analysis, meta-regression of 21 randomized trials and GRADE. Diabetes Obes. Metab. 20(8), 2009-2013 (2018)

14. Zhao, J., Freeman, B., Li, M.: Can mobile phone apps influence people's health behavior change? An evidence review. J. Med. Internet Res. 18(11), 1-15 (2016)

15. Deacon, A.J., Chee, J.J., Chang, W.J.R., Harbourne, B.A.: Mobile applications for diabetes mellitus self-management: a systematic narrative analysis. In: Successes and Failures in Telehealth Conference, SFT-2017, pp. 17-30 (2017)

16. Venkatesh, V., Morris, M.G., Davis, G.B., Davis, F.D.: User acceptance of information technology: toward a unified view. MIS Q. 27(3), 425-478 (2003)

17. Petersen, F., Pather, S., Tucker, W.D.: User acceptance of ICT for diabetes self-management in the Western Cape, South Africa. In: African Conference of Information Systems and Technology (ACIST), pp. 1-11 (2018)

18. Nelson, L.A., Mulvaney, S.A., Gebretsadik, T., Ho, Y.X., Johnson, K.B., Osborn, C.Y.: Disparities in the use of a mHealth medication adherence promotion intervention for lowincome adults with type 2 diabetes. J. Am. Med. Inform. Assoc. 23(1), 12-18 (2016)

19. Hoque, R., Sorwar, G.: Understanding factors influencing the adoption of mHealth by the elderly: an extension of the UTAUT model. Int. J. Med. Inform. 101, 75-84 (2017) 
20. Müller, A.M.: Behavioural mHealth in developing countries: what about culture? Eur. Health Psychol. 18(6), 294-296 (2016)

21. Singh, K., et al.: Many mobile health apps target high-need, high-cost populations, but gaps remain. Health Aff. 35(12), 2310-2318 (2016)

22. GSMA: South Africa mHealth Landscape (2013). http://www.gsma.com/mobilefordevelo pment/wp-content/uploads/2013/08/South-Africa-mHealth-Landscape_June-2013.pdf. Accessed 15 Sept 2015

23. Aranda-Jan, C.B., Mohutsiwa-Dibe, N., Loukanova, S.: Systematic review on what works, what does not work and why of implementation of mobile health (mHealth) projects in Africa. BMC Public Health 14(1), 188 (2014)

24. Scheibe, M., Reichelt, J., Bellmann, M., Kirch, W.: Acceptance factors of mobile apps for diabetes by patients aged 50 or older: a qualitative study. Med. 2.0 4(1), e1 (2015)

25. Zhang, Y., et al.: Factors influencing patients' intentions to use diabetes management apps based on an extended unified theory of acceptance and use of technology model: web-based survey. J. Med. Internet Res. 21(8), e15023 (2019)

26. Wolff-Piggott, B., Coleman, J., Rivett, U.: The clinic-level perspective on mHealth implementation: a South African case study. Inf. Technol. Dev. 24(3), 532-553 (2018)

27. Macdonald, E.M., Perrin, B.M., Kingsley, M.I.: Enablers and barriers to using two-way information technology in the management of adults with diabetes: a descriptive systematic review. J. Telemed. Telecare 24, 319-340 (2018)

28. Dadgar, M., Joshi, K.D.: The role of information and communication technology in selfmanagement of chronic diseases: an empirical investigation through value sensitive design. J. Assoc. Inf. Syst. 19(2), 86-112 (2018)

29. Statistics South Africa: General Household Survey. Statistical release P0318, May 2018

30. Petersen, F., Brown, A., Pather, S., Tucker, W.D.: Challenges for the adoption of ICT for diabetes self-management in South Africa. Electron. J. Inf. Syst. Dev. Ctries. 1-14 (2019). https://doi.org/10.1002/isd2.12113

31. GSMA: The Mobile Economy: Africa 2016 (2016)

32. Department of Health: mHealth Strategy 2015-2019. South Africa (2015)

33. Coetzer, J.: Application of HCI design principles in overcoming information illiteracy: case of a m-health application for a rural community in South Africa. In: 2018 International Conference on Intelligent and Innovative Computing Applications, ICONIC 2018, pp. 1-7 (2018)

34. Huang, Z., Soljak, M., Boehm, B.O., Car, J.: Clinical relevance of smartphone apps for diabetes management: a global overview. Diabetes Metab. Res. Rev. 34(4), e2990 (2018)

35. Myers, M.D.: Qualitative research in information systems. MIS Q. 21(2), 241 (1997)

36. Saunders, M., Lewis, P., Thornhill, A.: Understanding research philosophies and approaches. Res. Methods Bus. Stud. 2009, 106-136 (2009)

37. Marshall, M.N.: Sampling for qualitative research. Family Pract. 13(6), 522-525 (1996)

38. Cresswell, J.W.: Research Design: Qualitative, Quantitative, and Mixed Methods Approaches, 3rd edn. SAGE Publications, Thousand Oaks (2014)

39. Kleine, D.: ICT4WHAT?-Using the choice framework to operationalise the capability approach to development. J. Int. Dev. 22(5), 674-692 (2010)

40. Quaglio, G.L., et al.: Information and communications technologies in low and middleincome countries: survey results on economic development and health. Health Policy Technol. 5(4), 318-329 (2016)

41. Ahmed, T., Lucas, H., Khan, A.S., Islam, R., Bhuiya, A., Iqbal, M.: eHealth and mHealth initiatives in Bangladesh: a scoping study. BMC Health Serv. Res. 14(1), 260 (2014) 
42. Akhlaq, A., McKinstry, B., Bin Muhammad, K., Sheikh, A.: Barriers and facilitators to health information exchange in low- and middle-income country settings: a systematic review. Health Policy Plan. 31(9), 1310-1325 (2016)

43. Kenny, G., O’Connor, Y., Eze, E., Ndibuagu, E., Heavin, C.: A ground-up approach to mHealth in Nigeria: a study of primary healthcare workers' attitude to mHealth adoption. Procedia Comput. Sci. 121, 809-816 (2017)

44. Health Systems Trust: South African Health Review 2013/2014 (2014)

45. Department of Health: mHealth Strategy. South Africa (2015)

46. Rogers, E.M.: Diffusion of Innovations. Free Press, New York (2003) 